\title{
Query Sense Discovery Approach to Realize the User's Search Intent
}

\author{
Tarek Chenaina, College of Computer Science and Engineering, Taibah University, Yanbu, Saudi Arabia \\ Sameh Neji, Faculty of Economics and Management, Sfax University, Tunisia \\ Abdullah Shoeb, Faculty of Computers and Information, Fayoum University, Egypt
}

\begin{abstract}
The main goal of information retrieval is getting the most relevant documents to a user's query. So, a search engine must not only understand the meaning of each keyword in the query but also their relative senses in the context of the query. Discovering the query meaning is a comprehensive and evolutionary process; the precise meaning of the query is established as developing the association between concepts. The meaning determination process is modeled by a dynamic system operating in the semantic space of WordNet. To capture the meaning of a user query, the original query is reformulated into candidate queries by combining the concepts and their synonyms. A semantic score characterizing the overall meaning of such queries is calculated, and the one with the highest score was used to perform the search. The results confirm that the proposed "query sense discovery" approach provides a significant improvement in several performance measures.
\end{abstract}

\section{KEYWORDS}

Information Retrieval, Query Reformulation, Search Engine, Semantic Search, WordNet

\section{INTRODUCTION}

The major objective of Information Retrieval (IR) systems is to find relevant documents for a user's query (Grechanik et al., 2010; Zhai et al., 2015) Many IR systems are based on the traditional bag of words (BOW) approach. The different meanings of the query keywords are not taken into consideration, leading to an ambiguity caused by the polysemy. In most cases, words that contained into a query are polysemous. It is often possible to understand the meaning of a word from the set of words which used within; this is the notion of context. For example, the word "note" may mean "a notation representing the pitch and duration of a musical sound", "a brief written record", or "a piece of paper money". Disambiguation lies into the capacity of the system to exhibit relevant synonyms of the concept i.e. to determine the precise sense that the concept has in the query context.

To solve the problem of query disambiguation, several works have been done (ALMasri et al., 2016; Fernández-Reyes et al., 2018; Serizawa and Kobayashi, 2013). In order to retrieve the truly relevant documents, majority of works on disambiguation (Hirst et al., 1998; Khan and Feng Luo, 2003; Mihalcea and Moldovan, 2000) addresses the problem by measuring the similarity between the initial query and the documents. This method is not optimal, it is necessary to proceed with disambiguation of the query independently of the document, because the ambiguity intrinsically linked to the concepts of this query degrades the search effectiveness. 
Recent works (Bobed and Mena, 2016; Yan et al., 2017; Zingla et al., 2016) on the "query expansion" add similar terms, from those initially used. These terms are suggested either from resulting documents from the original query (blind expansion, relevance feedback, etc.) from a linguistic resource or from the query logs. Applying these approaches leads into a risk of introducing a noise in the search results (query drift) which yields a deviation from the user's intention. The first approach suffers from large size of the Web resources that degrades the approach effectivity. Moreover, these approaches do not contribute getting closer to the user's desired meaning because of the disambiguation does not focus only on the original terms of the query which obstructs the process of discovering the meaning of the query. Indeed, the lack of understanding of the factors influence the query's meaning and the results they produce because of the effect of relative positions of the words. This is due to the interrelationship of several parameters such as the dispersion of the concepts on the branches of the ontology and their depth and the semantic similarity between the concepts and their predecessors.

In this work, the case of a user requiring information on a specific topic through a query (ad-hoc search) is studied. One of the main problems regarding this search type is detection of query meaning subject to information user's need. As WordNet (George A. Miller, 1995) is one of the well-known and widely used external information resources that has been used in the proposed approach. WordNet provides a conceptual framework for the structured representation of query's context, in which nouns, verbs, adjectives and adverbs are organized by a variety of semantic relationships. Each concept has a set of synsets (synonyms) that represent its sense.

The next sections are organized as follows. Section 2 presents the related works on using semantic search in IR. Section 3 introduces the details of the main contribution "the discovery of the meaning of the query" (QSD). An evaluation of the results with the INEX2009 test collection is presented and discussed in section 4 using many metrics: precision, recall, Mean Average Precision (MAP), GMAP, $\mathrm{R}_{\text {prec}}$, Mean Reciprocal Rank (MRR), P@n, Recall Oriented Measure (ROM) and PRES (Patent Retrieval Evaluation Score). Finally section 5 summarizes the ideas and findings that are presented in this paper.

\section{RELATED WORKS}

The more knowledge content grows on the Internet the more effective search tools will be needed. The traditional (keywords) search is becoming insufficient to the searchers (Agrawal et al., 2013; Zhu et al., 2017). $41 \%$ of traditional search engines users are reported conflicting or contradictory search results unable to find the correct information and $34 \%$ of them are found that important information was missing from search results (Lee and Ma, 2012). Thus, the powerful search engines like Google, Yandex and Bing use semantic search ideas but the details of underlying techniques are not publically described (Giceva and Sadoghi, 2018). Semantic search is the next generation search paradigm after traditional one (Tablan et al., 2015). Semantic search approaches are hard to classify due to the great number of dimensions involved in this field (Sánchez Fernández, 2009). Hundreds of research articles have been conducted in a wide range of semantic search systems. Topics like query expansion (QE), word sense disambiguation (WSD), question answering (QA), word embeddings (WE), and named entity disambiguation (NED) are examples of such systems.

High quality retrieved data is the precious goal of any search engine. QE is classified as one of the most effective techniques to enhance the retrieval (Khennak et al., 2016). QE process, in its broad meaning, consists of adding new keywords to the original query. Pseudo-relevance feedback (PRF) is a method that used in a branch of automatic query modification technique. PRF assumes that the initial retrieved documents are relevant and then it uses these documents to find more relevant terms to the query or it just re-weighs the original query terms (Karisani et al., 2016). Word embeddings (WE) is a common name to a set of techniques to model languages and extract interested features. In WE, each word is represented as a real-valued vector (feature). In this model, the similarity of some words' contexts implies the closeness of these words' vectors (Fernández-Reyes et al., 2018). 
WE has been proved its capability to pick up the lexical semantics due to its power in capturing the word-similarity and relevance (Noraset et al., 2016). NED is another application of semantic search with mission of correctly map entity's mention, in a text, to its record in a knowledge base (Wang et al., 2017). Last application to introduce here is QA, its purpose is discovering similar previous questions in the light of a new question because answers of the existing similar questions are nominated to be answers for the new question (Othman et al., 2017). Table 1 summarizes recent works in the mentioned fields of semantic search systems.

Table 1. Recent works in different fields of semantic search

\begin{tabular}{|c|c|c|c|}
\hline Research & Topic & Used tools/techniques & Scope \\
\hline $\begin{array}{l}\text { - (Goslin and Hofmann, 2018) } \\
\text { - (Zingla et al., 2016) } \\
\text { - (Yan et al., 2017) } \\
\text { - (Latard et al., 2017) }\end{array}$ & $\mathrm{QE}$ & $\begin{array}{l}\text { - Wikipedia } \\
\text { - Wikipedia \& DBpedia } \\
\text { - Semantic similarity } \\
\text { - Semantic connections }\end{array}$ & $\begin{array}{l}\text { - general } \\
\text { - Tweets } \\
\text { - few/empty results retrieved } \\
\text { - directed to scientific search }\end{array}$ \\
\hline $\begin{array}{l}\text { - (Dahir et al., 2018) } \\
\text { - (Singh and Sharan, 2018) } \\
\text { - (Audeh et al., 2017) }\end{array}$ & PRF & $\begin{array}{l}\text { - top } 10 \text { retrieved documents } \\
\text { - filter \& genetic algorithm } \\
\text { - PRF \& ontology }\end{array}$ & $\begin{array}{l}\text { - general } \\
\text { - general } \\
\text { - general }\end{array}$ \\
\hline $\begin{array}{l}\text { - (Kim et al., 2017) } \\
\text { - (Fernández-Reyes et al., 2018) } \\
\text { - (Stein et al., 2019) } \\
\text { - (Othman et al., 2017) } \\
\text { - (Habibi et al., 2016) }\end{array}$ & WE & $\begin{array}{l}\text { - "Word Mover Distance" } \\
\text { - word2vec } \\
\text { - word2vec \& fastText } \\
\text { - cosine similarity } \\
\text { - WordNet \& word2vec }\end{array}$ & $\begin{array}{l}\text { - general } \\
\text { - general } \\
\text { - general } \\
\text { - community question answering } \\
\text { - QA }\end{array}$ \\
\hline $\begin{array}{l}\text { - (Wang et al., 2017) } \\
\text { - (Chen et al., 2018) } \\
\text { - (Zhao et al., 2016) }\end{array}$ & NED & $\begin{array}{l}\text { - unsupervised learning \& Wikipedia } \\
\text { - probabilistic model } \\
\text { - Wikipedia }\end{array}$ & $\begin{array}{l}\text { - community question answering } \\
\text { - general } \\
\text { - general }\end{array}$ \\
\hline
\end{tabular}

The current work intersects with many other researches in the area of QE; it is gaining the advantages of using QE like precision increasing and suitability for the novice user. Many researches suffer from the intrinsic weakness in QE, namely query drift and not locating the positions of new keywords correctly which the proposed approach avoids as shown in the next section.

\section{QUERY SENSE DISCOVERY APPROACH}

The proposed approach is focus on calculating the global meaning of the query. The different senses of a concept are represented by regions in a semantic space in WordNet with a distance. The process of determining the precise sense in a given context is modeled by a dynamic system operating on that space. Using WordNet makes it possible to implement this model and thus to realize an operational disambiguation tool.

The main motivation of current work, Query Sense Discovery (QSD) approach, is to improve the relevance of results returned by a search engine. The objectives consist providing, on the one hand, clarifications concerning the understanding of the user's search intent. On the other hand, explain the parameters involved in the calculation of the global meaning of the query, and judge the relevance and relative interest of such parameters. These parameters allow more closeness to the meaning desired by the user. Figure 1 presents QSD in five steps.

\section{Lexical Analysis of the Query}

Lexical analysis consists of decomposing the query into lexical units that are characterized by their grammatical classes (noun, verb, adverb, and adjective). Indeed, a lexical unit can take several 


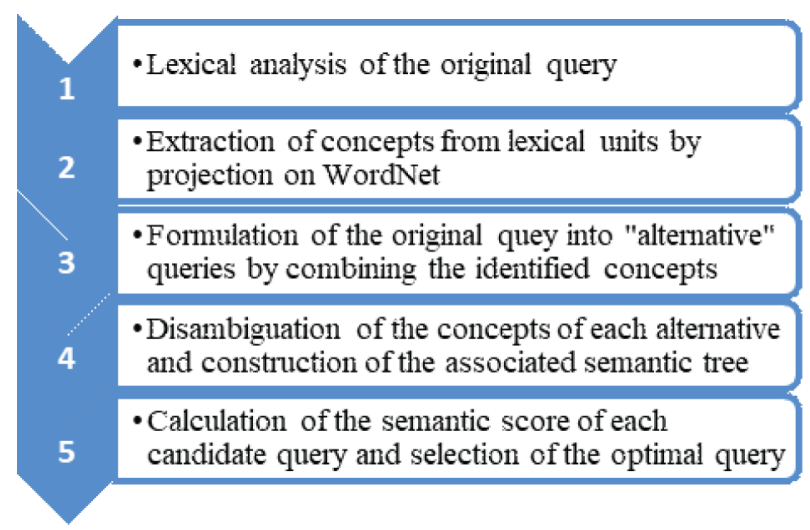

meanings depending on its grammatical class. To define the grammatical structure of the query, the "Stanford Parser" (Klein and Manning, 2007) is used. The following example illustrates the importance of defining the grammatical class of query's keywords leading to the concepts' associated meaning. For example, in the two following queries Q1 and Q2, the word "nice" can have several senses depending on its grammatical class. Nice, as an adjective, describes a beautiful thing, but Nice, as a noun, points to a city in France.

$$
\begin{aligned}
& \text { Q1: } \underbrace{\text { Qy photo }}_{\text {Noun }} \underbrace{\text { Nice }}_{\text {Verb }} \underbrace{\text { is }}_{\text {Verb }} \underbrace{\text { beautiful }}_{\text {Adjective }} \\
& \text { Adice }
\end{aligned}
$$

\section{Extracting the Concepts of the Query}

After the lexical analysis of the query, QSD proceeds by detecting the concepts (mono or multi-word) included in WordNet. The decomposition of the query into concepts is carried out by concatenating only the adjacent words belonging to the same grammatical class (tag) to preserve the meaning of the query. Then, the stop-words are deleted to avoid noise. A lemmatization by the Porter algorithm (Porter, 2001, 1980) is carried out in the search phase to reduce the searched words to their lemmas.

The following example illustrates how to extract the concepts of the query "The ear-noseand-throat doctor attains a training course in neural networks". The concepts that do not belong to WordNet (surrounded by dotted lines) will not be considered later. Figure 2 shows that 11 concepts, surrounded by oval shape solid lines, have been identified in WordNet. The candidate queries are then formulated from the identified concepts. 


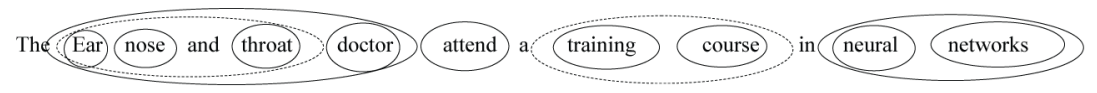

\section{Formulation of Alternative Queries}

Figures 3 and 4 explain the "Generate" algorithm that formulates alternative queries using the concepts identified in previous section. It proceeds by a rational reorganization of the identified concepts keeping the same lexical structure of the original query which leads to a set of candidate queries. Each query is characterized by the meaning derived from the context of the identified concepts. "Generate" organizes the candidate queries in a tree structure.

Figure 5 illustrates the alternative queries derived from the original query "prescription drug user interface". The query alternatives are determined by traversing the tree from root to all leaves in left-right fashion. In this example there are eight possible candidates. In the general case, number of alternatives is equals to $2^{\text {(query_size-1) }}$. Table 2 lists its candidate queries in the same order displayed in the top-down view of the tree. It also shows many possible senses of the query concepts.

Table 2 shows the importance of formulating different alternatives of identified concepts; each candidate query is characterized by a global meaning different from the other. A question that arises naturally is "what is the best query among candidate queries that returns more relevant documents to the top of the retrieved list?"

\section{Construction of Semantic Tree of a Candidate Query}

Each candidate query is represented as a semantic tree. Figure 6 presents an example of a semantic tree whose leaves represent the query's concepts. The construction of the tree is performed after calculating the sense of each concept using (Eq.1). QSD uses the WSD procedure (Fodeh et al., 2011) taking into consideration all the concept's senses. Indeed, the global meaning is decided with reference to the senses of all the query's concepts. Moreover, the correlation that connects the senses of the concepts imposes a more global reasoning of all the actors (the concepts). Let $S_{i}=\left\{s_{i 1}, s_{i 2}, \ldots, s_{i k}\right\}$ be the set of all senses associated with the concept $C_{i}$ according to WordNet. The most appropriate sense $\hat{s}_{i}$ of the concept $C_{i}$ is the sum of its similarity with the senses of the other query's concepts:

$$
\hat{s}_{i}=\arg \max _{s_{i l} \in S_{i}} \sum_{t_{j} \in q} \max _{s_{j m} \in S_{j}} \delta\left(s_{i l}, s_{j m}\right)
$$

where $\delta\left(s_{p}, s_{q}\right)$ is the WuAndPalmer similarity between two senses $s_{p}$ and $s_{q}$.

From the original query "olive oil health benefit", the algorithm generates two candidate queries, see Figure 6, Q1 that has four concepts (in bold) while Q2 that has three concepts (in italics). The semantic tree is then constructed for all candidate queries by projection the concepts on WordNet. For reasons of visibility, the two semantic trees are merged into a single one.

It is neither the concepts, nor their senses, but their uses and relative meanings are the source of query misunderstanding. Table 3 shows that the concept "olive" has five senses: a natural fruit, a tree, a wood, a fruit to savor and a color. In the presence of the concepts "oil", "health", and "benefit", the best calculated sense is "natural fruit". However, in the case of the "olive oil" concept, the best calculated sense is "oil from olives" which associates the notion of "benefit of olive oil". 
Figure 3. Flowchart of "Generate" algorithm

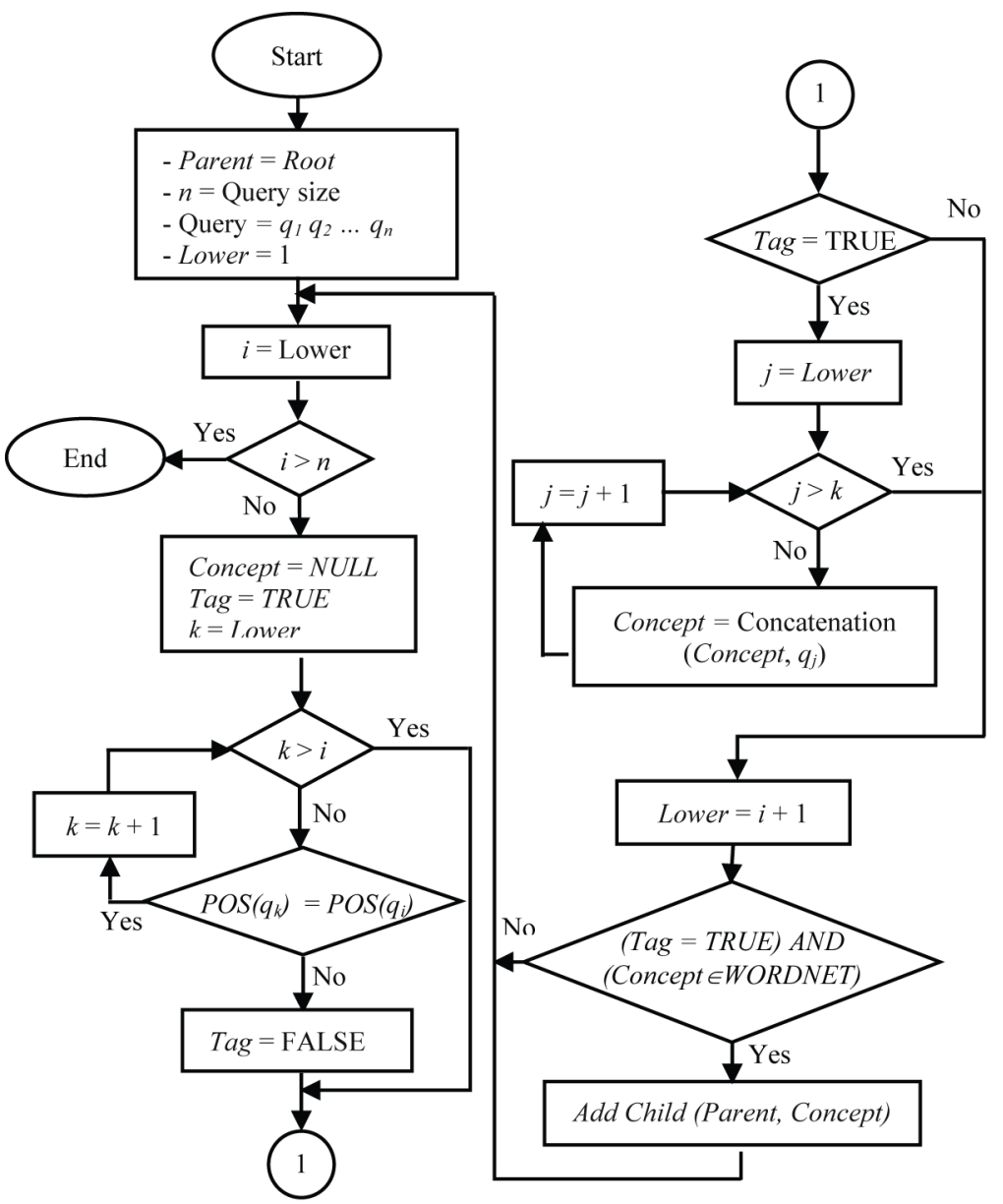

\section{Calculation of the Query's Meaning}

This step is dedicated for determining the optimal meaning of the original query by computing the semantic scores of the alternative queries. Equation (2) shows the proposed semantic scoring function:

$$
\text { Can_Score }{ }^{-1}=D^{\alpha} \sum_{i=1}^{n} \frac{1}{\left(\operatorname{depth}\left(c_{i}\right)\right)^{\beta}} \sum_{\substack{c=c_{i} \\ c \in \operatorname{path}\left(c_{i}, L C S\right)}} \log (1+(c, \text { hyper }(c)))
$$




\section{Figure 4. Pseudocode of "Generate" algorithm}

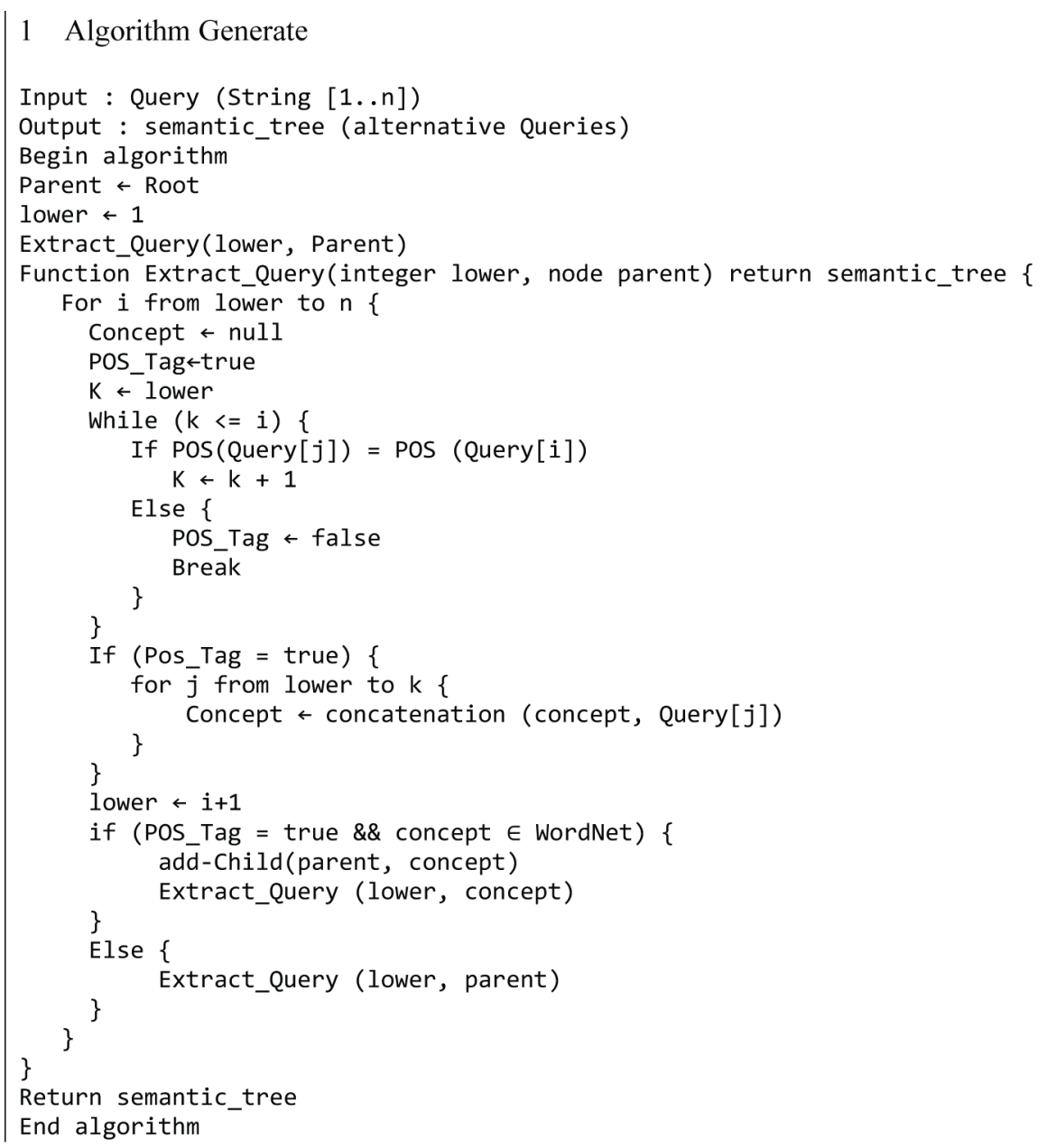

where, $n$ is the number of concepts in the query, $c_{i}$ is the $i^{\text {th }}$ concept, $L C S$ is the least common subsumer of $c_{1}, c_{2}, \ldots, c_{n}$, hyper (c) is the hypernym of concept " $c$ ", $\delta(c$, hyper $(c))$ is the semantic similarity between the concept " $c$ " and its hypernym, $D$ is number of branches containing at least one concept of the query, and $\operatorname{depth}\left(c_{i}\right)$ is depth of the concept up to LCS.

There are three notions affecting the global meaning of the query. Firstly, the dispersion of concepts in the semantic tree is inversely proportional to the precise meaning expected by the user. Indeed, as more scattering the concepts on distant branches as more they belong to divergent contexts. I.e. the lower dispersion factor $D$ reflects a better transparency of the meaning.

High values of $\alpha$ reflect the difficulty of identifying the common context of the different concepts. It will therefore be difficult to select the precise meaning of the query, which influences the accuracy of the results and the thematic homogeneity of the retrieved documents. Furthermore, the dispersion coefficient $\alpha$ provide the possibility to customize the search results according to the level of user expertise. Indeed, the candidate queries those have semantic scores with a high coefficient $\alpha$ return general documents from various contexts. This argument coincides with the expectations of a novice who wants to provide simple and general documents in separate themes. On the contrary, a low value is assigned to the coefficient $\alpha$ for an expert who looking for specific documents. In this case, the 


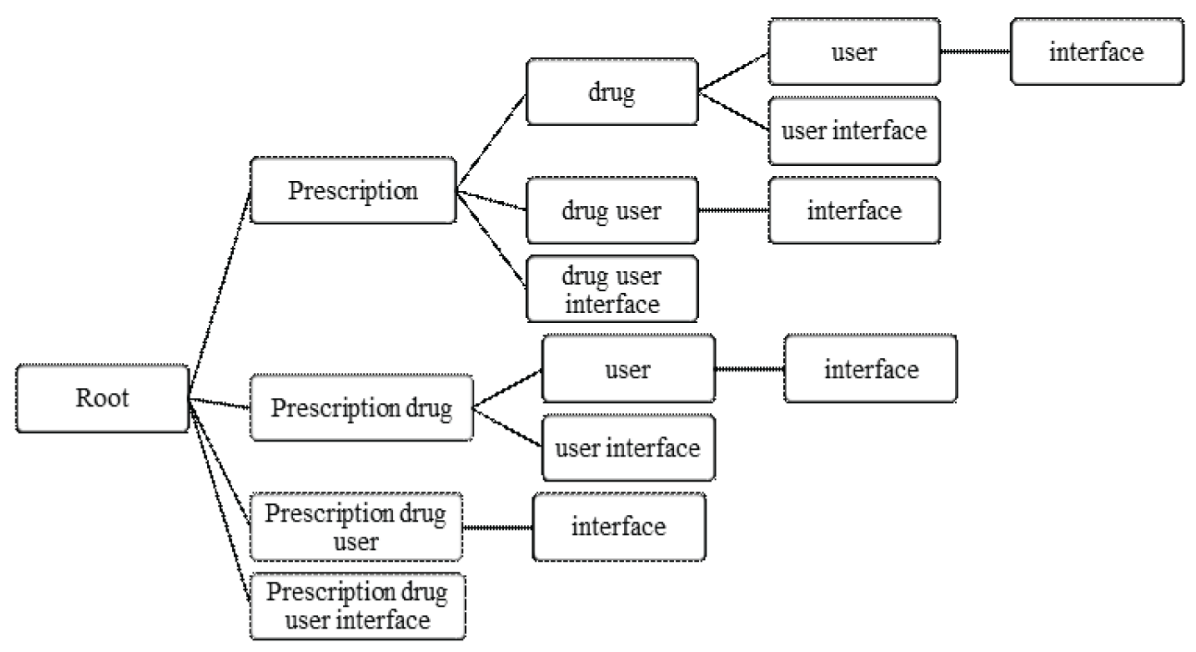

search is carried out on concepts belonging to very close branches or even on the same branch. Another observation is that the deeper the concept in the tree, the less abstract of its characterization. High values of $\beta$ make it possible to orient the search using more specific concepts belonging to deeper branches, thus returning specific documents in a precise context.

The third factor to consider, in calculating the semantic score, is the similarity between the current concept and the concept that it subsumes up to the least common subsumer (LCS) node, which is an ancestor of all the query's concepts $\left(c_{1}, c_{2}, \ldots, c_{n}\right)$. Let " $y$ " be a node that is descendant of a node " $x$ ". if $\exists x_{0}, x_{1}, \ldots, x_{p}$ such as: $x_{0}=\operatorname{LCS}$ of $c_{1}, c_{2}, \ldots, c_{n}$, and $x_{p}$ is the current concept, $\forall \mathrm{k} \in[1$, p], hypernym $\left(x_{\mathrm{k}}\right)=x_{\mathrm{k}-1}$. The Resnik method (Resnik, 1995) is used, it is based on the notion of informational content (IC) that defines the semantic similarity between two concepts by the amount of information they share.

\section{Interpreting the Extraction of Optimal Meaning by Example}

Before presenting the experimental results, it is better to present a concrete example shows the importance of the proposed approach and makes tangible the extraction of the query's optimal sense.

By continue with the query "prescription drug user interface", referring to Table 2, the concept "prescription" of the query \#4 refers to a rather general sense (the action of prescribing authoritative rules or directions) in the absence of other concepts. In query \#3, the concept "prescription" refers to the sense "prescription for an optician" in the presence of the concept "interface" refers to the meaning of a program controlling the display on a screen. Note that the concept "drug user" that expresses the notion of "a person who takes drugs" (Figure 7.a), lowers the semantic score. The meaning of query \#2 is becoming clearer; the concept "prescription" reflects the sense "medical prescription" in the presence of the concept "drug" which means a substance that is used as a medicine or narcotic. Finally, query \#6 (Figure 7.b) attains the best semantic score (table 4), hence it is optimal compared to the original query as it refers to the meaning of an information system of medical prescription.

The interpretation of the results in Table 4 asserts that meaning is an evolving contextual process; the precise meaning of the query is established as developing the association between concepts. The purpose of the QSD approach is to reproducing the senses as an act of association between concepts. 
Table 2. Query alternatives for the query "prescription drug user interface"

\begin{tabular}{|c|c|c|c|}
\hline No. & Alternative query & $\begin{array}{c}\text { \# of } \\
\text { concepts }\end{array}$ & Concept sense * \\
\hline 1 & $\begin{array}{l}\text { Prescription drug } \\
\text { user interface }\end{array}$ & 4 & $\begin{array}{l}\text { prescription: a drug that is available only with written instructions } \\
\text { from a doctor or dentist to a pharmacist } \\
\text { drug: a substance that is used as a medicine or narcotic } \\
\text { user: a person who makes use of a thing; someone who uses or } \\
\text { employs something } \\
\text { interface: a surface forming a common boundary between two } \\
\text { things }\end{array}$ \\
\hline 2 & $\begin{array}{l}\text { Prescription drug } \\
\text { (user interface) }\end{array}$ & 3 & $\begin{array}{l}\text { prescription: a drug that is available only with written instructions } \\
\text { from a doctor or dentist to a pharmacist } \\
\text { drug: a substance that is used as a medicine or narcotic } \\
\text { (user interface): a program that controls a display for the user } \\
\text { (usually on a computer monitor) and that allows the user to interact } \\
\text { with the system }\end{array}$ \\
\hline 3 & $\begin{array}{l}\text { Prescription (drug } \\
\text { user) interface }\end{array}$ & 3 & $\begin{array}{l}\text { prescription: written instructions for an optician on the lenses for a } \\
\text { given person } \\
\text { (drug user): a person who takes drugs } \\
\text { interface: a program that controls a display for the user (usually } \\
\text { on a computer monitor) and that allows the user to interact with the } \\
\text { system }\end{array}$ \\
\hline 4 & $\begin{array}{l}\text { Prescription (drug } \\
\text { user interface) }\end{array}$ & 2 & $\begin{array}{l}\text { prescription: directions prescribed beforehand; the action of } \\
\text { prescribing authoritative rules or directions } \\
\text { (drug user interface): None }\end{array}$ \\
\hline 5 & $\begin{array}{l}\text { (Prescription } \\
\text { drug) user } \\
\text { interface }\end{array}$ & 3 & $\begin{array}{l}\text { (prescription drug): a drug that is available only with written } \\
\text { instructions from a doctor or dentist to a pharmacist } \\
\text { user: a person who makes use of a thing; someone who uses or } \\
\text { employs something } \\
\text { interface: computer circuit consisting of the hardware and } \\
\text { associated circuitry that links one device with another }\end{array}$ \\
\hline 6 & $\begin{array}{l}\text { (Prescription } \\
\text { drug) (user } \\
\text { interface) }\end{array}$ & 2 & $\begin{array}{l}\text { (prescription drug): a drug that is available only with written } \\
\text { instructions from a doctor or dentist to a pharmacist } \\
\text { (user interface): a program that controls a display for the user } \\
\text { (usually on a computer monitor) and that allows the user to interact } \\
\text { with the system }\end{array}$ \\
\hline 7 & $\begin{array}{l}\text { (Prescription drug } \\
\text { user) interface }\end{array}$ & 2 & $\begin{array}{l}\text { (prescription drug user): None } \\
\text { interface: a surface forming a common boundary between two } \\
\text { things (two objects or liquids or chemical phases }\end{array}$ \\
\hline 8 & $\begin{array}{l}\text { (Prescription drug } \\
\text { user interface) }\end{array}$ & 1 & (prescription drug user interface): None \\
\hline
\end{tabular}

* These concept senses are excerpted from WordNet 3.0

\section{EXPERIMENTATION AND DISCUSSION}

INEX 2009 web collection was used to evaluate QSD (Query Sense Discovery) approach. This collection is characterized by the variety of its query lengths. Indri index builder was used to index the collection using Krovetz stemmer. The collection that contains 2,666,190 documents was processed by 57 queries. QSD is examined against many frameworks. Okapi, the classical keyword indexing was used as a. The second case was the query likelihood language model (Ponte and Croft, 1998), denoted as QL. QL was smoothed by Dirichlet method. QSD is also compared to SMERA and RM3 (Audeh et al., 2017). Table 5 shows the values of the underlying parameters. 
Figure 6. Merged semantic trees of alternative queries Q1 and Q2

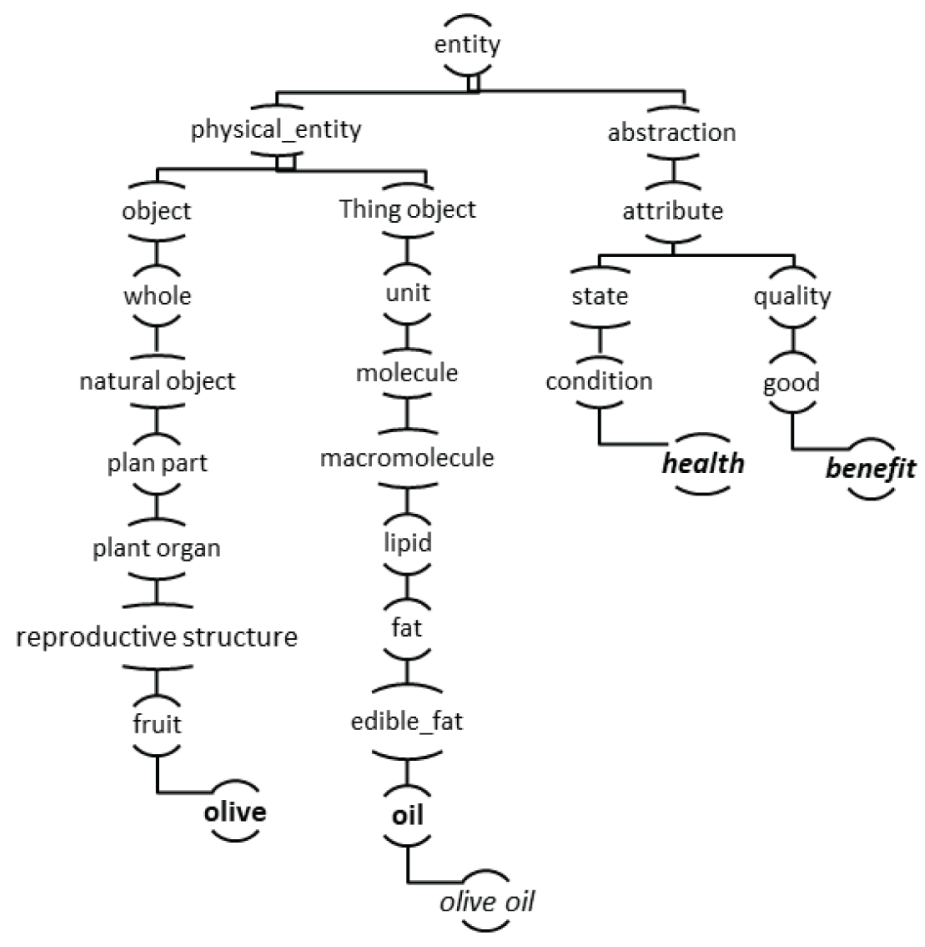

Table 3. Number of senses for Q1 and Q2

\begin{tabular}{|l|l|l|l|l|l|}
\hline \multicolumn{1}{|c|}{ Concepts } & \multicolumn{1}{|c|}{ Olive } & \multicolumn{1}{c|}{ Oil } & \multicolumn{1}{c|}{ Health } & Benefit & (Olive Oil) \\
\hline \# of sense's concept & 5 & 3 & 2 & 3 & 1 \\
\hline \# of possible senses & $\begin{array}{l}\text { Q1: } 5 \times 3 \times 2 \times 3=90 \\
\text { Q2: } 1 \times 2 \times 3=6\end{array}$ \\
\hline
\end{tabular}

Table 4. Semantic scores of candidate queries

\begin{tabular}{|l|l|}
\hline \multicolumn{1}{|c|}{ \# query } & \multicolumn{1}{c|}{ Can_Score } \\
\hline 1 & $4.861 \mathrm{E}-02$ \\
\hline 5 & $6.164 \mathrm{E}-02$ \\
\hline 2 & $1.464 \mathrm{E}-01$ \\
\hline 3 & $6.743 \mathrm{E}-02$ \\
\hline 6 & $1.878 \mathrm{E}-01$ \\
\hline
\end{tabular}




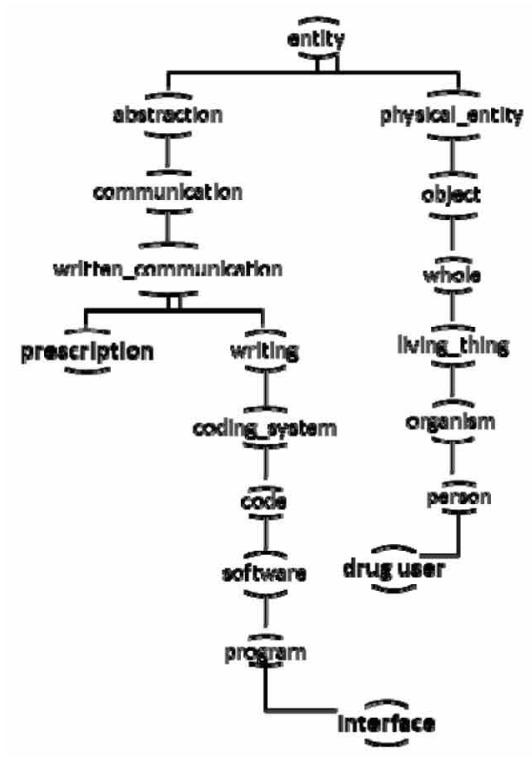

(a)

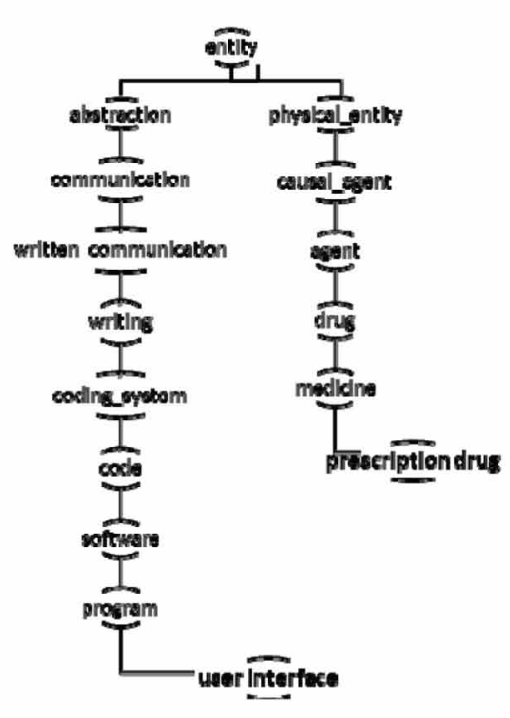

(b)

Table 5. Parameter values for the implemented approaches

\begin{tabular}{|l|l|}
\hline \multicolumn{1}{|c|}{ Parameter } & \multicolumn{1}{c|}{ Value } \\
\hline$k_{1}$ & 1.2 \\
\hline$K_{3}$ & 7 \\
\hline$b$ & 0.75 \\
\hline$\mu$ & 2500 \\
\hline
\end{tabular}

\section{Experiment 1}

Table 6 shows the obtained values of MAP, GMAP and precision of the first R documents where R is the number of relevant documents $\left(\mathrm{R}_{\text {prec }}\right)$ for the three approaches. Figure 8 depicts the comparison between them. Table 7 presents the improvements of approaches couples. By analyzing the results, the great enhancement was obtained when moving from baseline to QSD. It can be seen that the QSD approach has improved the GMAP by $11.8 \%$ compared to the QL model. The $\mathrm{R}_{\text {prec }}$ measure represents the calculated precision for the $\mathrm{R}_{\mathrm{th}}$ relevant document returned. The QSD model has improved the $\mathrm{R}_{\text {prec }}$ by $4.9 \%$ over the QL model. This improvement indicates that the proposed approach retrieves

Table 6. Results of MAP, GMAP, $\mathbf{R}_{\text {prec }}$

\begin{tabular}{|l|l|l|l|}
\hline & \multicolumn{1}{|c|}{ MAP } & \multicolumn{1}{c|}{ GMAP } & \multicolumn{1}{c|}{$\mathbf{R}_{\text {prec }}$} \\
\hline Baseline & 0.283 & 0.215 & 0.316 \\
\hline QL & 0.330 & 0.272 & 0.356 \\
\hline QSD & 0.354 & 0.304 & 0.373 \\
\hline
\end{tabular}


Table 7. Improvements of MAP, GMAP, $\mathbf{R}_{\text {prec }}$

\begin{tabular}{|l|l|l|l|}
\hline & \multicolumn{1}{|c|}{ MAP } & \multicolumn{1}{c|}{ GMAP } & \multicolumn{1}{c|}{ R $_{\text {prec }}$} \\
\hline QSD over Baseline (\%) & 24.874 & 41.388 & 17.877 \\
\hline QSD over QL (\%) & 7.141 & 11.824 & 4.906 \\
\hline QL over Baseline (\%) & 16.551 & 26.438 & 12.364 \\
\hline
\end{tabular}

Figure 8. Comparison of MAP, GMAP, and $R_{\text {prec }}$

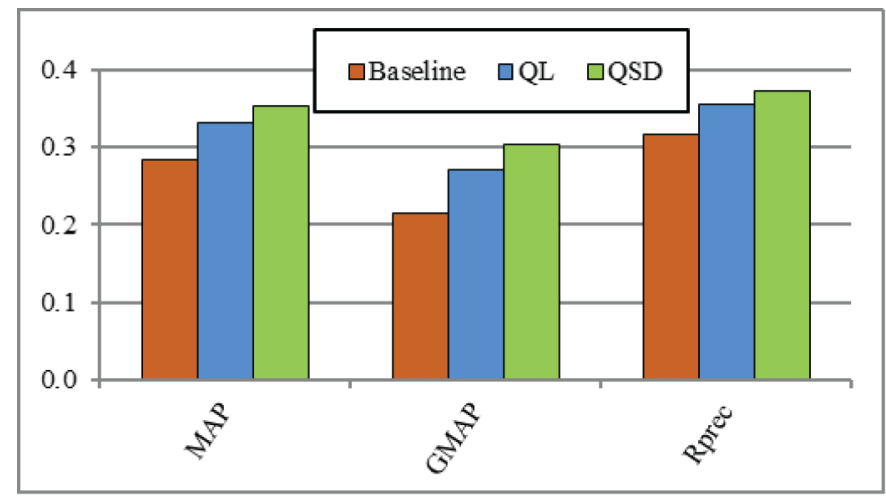

more relevant documents in the top ranked documents. The MAP has the advantage of estimating the overall performance of a search system. This is the most widely used metric in the field of IR. It takes into account the value of the average precision calculated for each tested query. QSD has improved the MAP by $7.1 \%$ compared to QL. This shows that QSD system is the most efficient.

\section{Experiment 2}

Second experiment was concentrated on Mean Reciprocal Rank (MRR), Patent Retrieval Evaluation Score (PRES), Recall, and Recall Oriented Measure (ROM). Table 8 presents the obtained values of the interested measures. Figure 9 compares the three approaches and Table 9 summarizes the improvements of the measures. It shows that QSD successes to improve MRR by 3.9\% over QL. MRR have great importance in this case because it reflects the rank at which the first relevant document is retrieved. PRES is another, relatively recent, measure that concentrates on the top ranked documents. QSD enhances PRES by 3\% over QL. QSD recall was enhanced by 1\% over QL. This is the least improvement in this table because the value of recall after 1000 retrieved documents is used, which means that almost all relevant documents were already retrieved regardless of the used approach. Also, recall is not the appropriate measure when focusing on the top ranked documents (Levene, 2011). ROM measure focuses on the last relevant retrieved document. QSD enhances ROM by 1.8\% over QL.

\section{Experiment 3}

The third experiment is dedicated to compare recall-precision graphs for the three approaches. Recallprecision graphs are of more importance as they provide useful details regarding the effectiveness of the retrieved documents ranking at different standard values of recall. The recall-precision measurement is equally dependent on both recall and precision. The effective information retrieval system should improve the recall-precision pair. 
Figure 9. Comparison of MRR, PRES, Recall, ROM

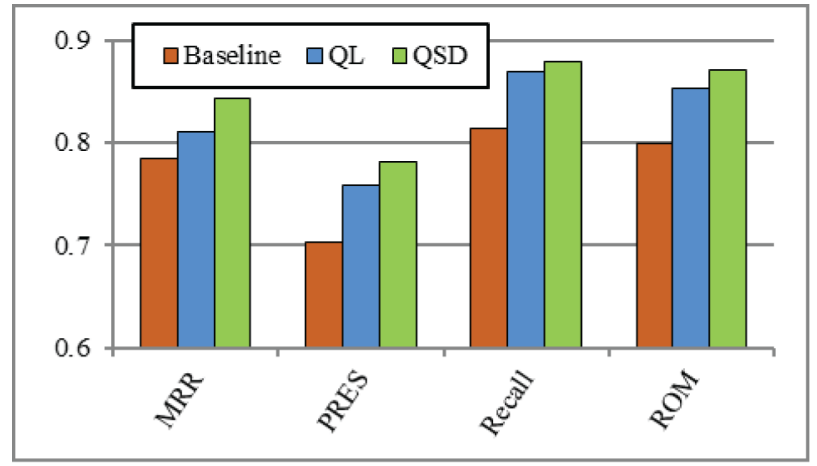

Table 8. Results of MRR, PRES, Recall, ROM

\begin{tabular}{|l|l|l|l|l|}
\hline & \multicolumn{1}{|c|}{ MRR } & \multicolumn{1}{c|}{ PRES } & \multicolumn{1}{c|}{ Recall } & \multicolumn{1}{c|}{ ROM } \\
\hline Baseline & 0.784 & 0.703 & 0.814 & 0.800 \\
\hline QL & 0.811 & 0.759 & 0.870 & 0.854 \\
\hline QSD & 0.843 & 0.782 & 0.879 & 0.870 \\
\hline
\end{tabular}

Table 9. Improvements of MRR, PRES, Recall, ROM

\begin{tabular}{|l|l|l|l|l|}
\hline & \multicolumn{1}{|c|}{ MRR } & \multicolumn{1}{c|}{ PRES } & \multicolumn{1}{c|}{ Recall } & \multicolumn{1}{c|}{ ROM } \\
\hline QSD over Baseline (\%) & 7.430 & 11.203 & 8.008 & 8.747 \\
\hline QSD over QL (\%) & 3.907 & 3.081 & 1.012 & 1.864 \\
\hline QL over Baseline (\%) & 3.390 & 7.878 & 6.926 & 6.758 \\
\hline
\end{tabular}

Figure 10. Average recall-precision graph

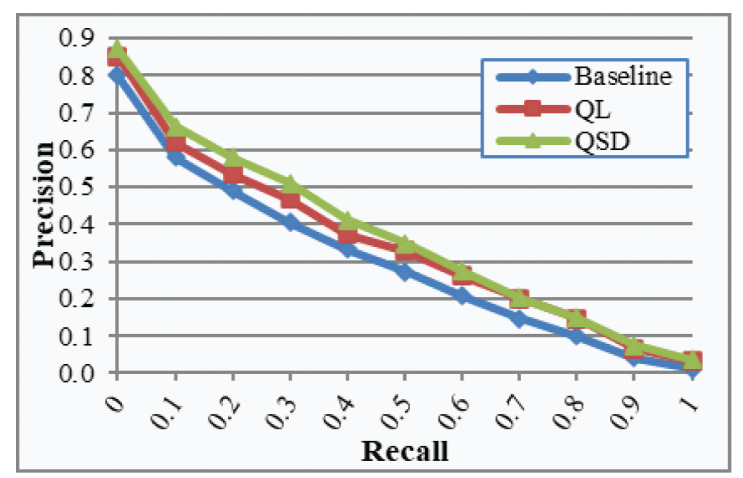


Figure 11. Precision @ different ranks

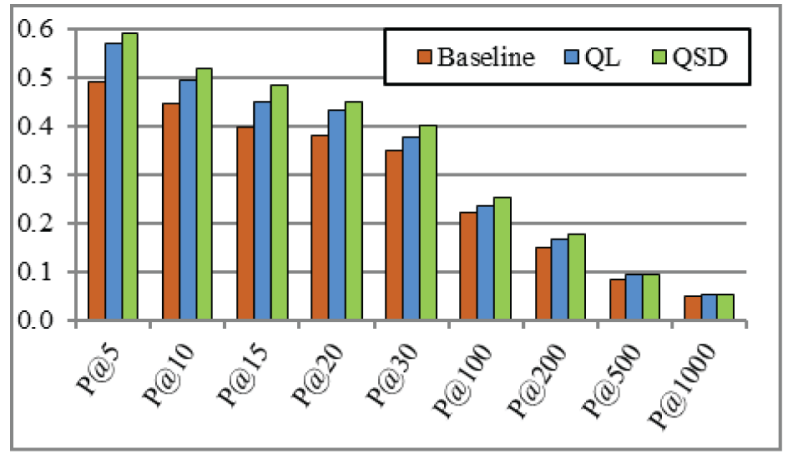

The behavior of the system varies depending on the precision and recall at the same time, i.e. the list of relevant documents and the rank of these documents as the recall measures the power of a system in retrieving all the relevant documents whereas the precision measures the power of how well it is doing at neglecting the non-relevant documents. Figure 10 shows the average recall-precision graph. It shows that QSD system outperforms the two other benchmark approaches.

\section{Experiment 4}

The fourth experiment compares precision@ $k$ to the three approaches, where $k=5,10,15,20,30$, $100,200,500,1000$. Figure 11 plots the precision at the mentioned ranks. As P@ $\mathrm{x}$ is the precision calculated in the $\mathrm{x}^{\text {th }}$ document returned, it's clearly observed from Figure 11 that QSD system surpasses the other two approaches for all these ranks of calculated precision. This means that QSD returns more of the relevant documents than the other two approaches.

\section{Experiment 5}

The fifth experiment compares results of QSD, SMERA, and RM3 as published in (Audeh et al., 2017). The three approaches, namely QSD, SMERA, and RM3, utilize INEX2009 to explore the approach capability. Table 10 shows the improvements of SMERA and RM3 as excerpted from (Audeh et al., 2017) and also the corresponding results of QSD. Figure 12 shows that QSD outperforms both of SMERA and RM3 in the mentioned measures.

Table 10. Improvements of MAP, P@10, MRR, and ROM in QSD, SMERA, and RM3

\begin{tabular}{|l|l|l|l|l|}
\hline & \multicolumn{1}{|c|}{ MAP } & \multicolumn{1}{c|}{ P@ 10 } & \multicolumn{1}{c|}{ MRR } & \multicolumn{1}{c|}{ ROM } \\
\hline QSD over QL (\%) & +7.14 & +4.98 & +3.91 & +1.86 \\
\hline SMERA over QL (\%) & +0.70 & -0.30 & +0.76 & +0.63 \\
\hline RM3 over QL (\%) & -0.32 & -0.76 & -0.37 & +0.04 \\
\hline
\end{tabular}




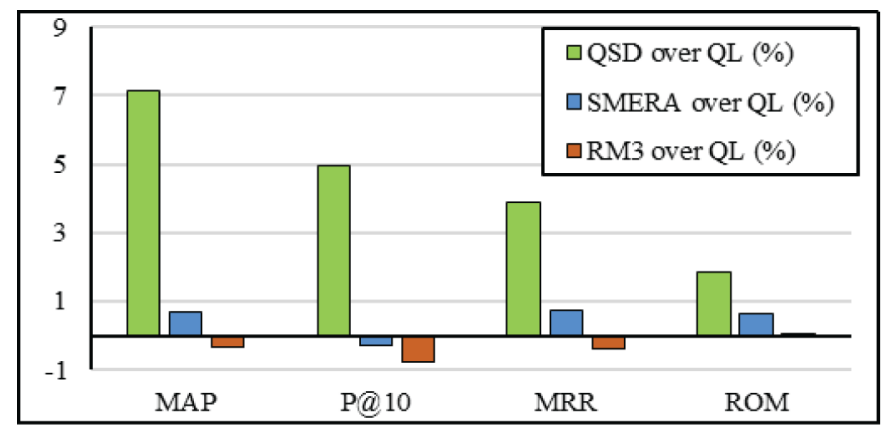

\section{CONCLUSION}

The proposed approach (QSD) is started by a lexical analysis to the query in order to keep the grammatical structure of its terms throughout the treatment process, due to its importance for the meaning computation. Secondly, an algorithm was proposed to generate "candidate" queries from the mono and multi-word concepts extracted from WordNet. Eventually, a novel semantic score was introduced to assess for each candidate query the closeness to user's search intent.

The semantic score utilizes many parameters that influencing the meaning of the query. The concepts' dispersion in the semantic network is inversely proportional to the meaning expected by the user; the lower the dispersion factor, the higher the transparency of meaning. Moreover, the depth of a concept, up to the least common subsumer, affects the meaning of the query as well as on the accuracy of the search results and the thematic homogeneity of the retrieved documents. The similarity between the concept and its parent is another parameter that influences the meaning of the query.

Query Sense Discovery (QSD) approach was improved MAP, GMAP and Rprec measures by $7.1 \%, 11.8 \%$ and $4.9 \%$, respectively compared to the QL approach. QSD has succeeded in increasing MRR by $3.9 \%$. QSD was improved P@ 10 by 4.98\%. The ROM measure is improved by QSD by $1.86 \%$ with respect to QL. Moreover, QSD improves the PRES measure by $3 \%$ and $11.2 \%$ respectively with respect to QL and Baseline.

Many research points can be improved in the future. The effect of reweigh some query's concepts according to their importance or position may be studied. Also, the used formula to disambiguate word sense may be developed to enhance the results. The semantic score formula may be enriched by more parameters that affects the meaning calculation. Other external resources, other than WordNet, may be used to provide more precise senses especially in the case of specific domain. 


\section{REFERENCES}

Agrawal, J., Sharma, N., Kumar, P., Parshav, V., \& Goudar, R. H. (2013). Ranking of searched documents using semantic technology. Procedia Engineering, 64, 1-7. doi:10.1016/j.proeng.2013.09.070

ALMasri, M., Berrut, C., \& Chevallet, J.-P. (2016). A Comparison of Deep Learning Based Query Expansion with Pseudo-Relevance Feedback and Mutual Information. The Southeast Asian Journal of Tropical Medicine and Public Health, 24(Suppl 1), 709-715. doi:10.1007/978-3-319-30671-1_57

Audeh, B., Beaune, P., \& Beigbeder, M. (2017). SMERA: Semantic mixed approach for web query expansion and reformulation. In Studies in Computational Intelligence (pp. 159-180). Springer., doi:10.1007/978-3-31945763-5_9

Bobed, C., \& Mena, E. (2016). QueryGen: Semantic interpretation of keyword queries over heterogeneous information systems. Inf. Sci. (Ny), 329, 412-433. doi:10.1016/j.ins.2015.09.013

Chen, J., Chen, Y., Zhang, X., Du, X., Wang, K., \& Wen, J. R. (2018). Entity set expansion with semantic features of knowledge graphs. Journal of Web Semantics, 52-53, 33-44. doi:10.1016/j.websem.2018.09.001

Dahir, S., El Qadi, A., \& Beiinis, H. (2018). Enriching user queries using DBpedia features and relevance feedback. Procedia Computer Science, 127, 499-504. doi:10.1016/j.procs.2018.01.148

Fernández-Reyes, F. C., Hermosillo-Valadez, J., \& Montes-y-Gómez, M. (2018). A Prospect-Guided global query expansion strategy using word embeddings. Information Processing \& Management, 54(1), 1-13. doi:10.1016/j. ipm.2017.09.001

Fodeh, S., Punch, B., \& Tan, P. N. (2011). On ontology-driven document clustering using core semantic features. Knowledge and Information Systems, 28(2), 395-421. doi:10.1007/s10115-010-0370-4

Giceva, J., \& Sadoghi, M. (2018). Hybrid OLTP and OLAP. In Encycl. Big Data Technol. Springer Int. Publ. doi:10.1007/978-3-319-63962-8_179-1

Goslin, K., \& Hofmann, M. (2018). A Wikipedia powered state-based approach to automatic search query enhancement. Information Processing \& Management, 54(4), 726-739. doi:10.1016/j.ipm.2017.10.001

Grechanik, M., Fu, C., Xie, Q., McMillan, C., Poshyvanyk, D., \& Cumby, C. (2010). A search engine for finding highly relevant applications. 10.1145/1806799.1806868

Habibi, M., Mahdabi, P., \& Popescu-Belis, A. (2016). Question answering in conversations: Query refinement using contextual and semantic information. Data \& Knowledge Engineering, 106, 38-51. doi:10.1016/j. datak.2016.06.003

Hirst, G., \& St-Onge, D. (1998). Lexical chains as representations of context for the detection and correction of malapropisms. WordNet An Electron. Lex. Database, 305, 305-332.

Karisani, P., Rahgozar, M., \& Oroumchian, F. (2016). A query term re-weighting approach using document similarity. Information Processing \& Management, 52(3), 478-489. doi:10.1016/j.ipm.2015.09.002

Khan \& Luo. (2003). Ontology construction for information selection. 10.1109/TAI.2002.1180796

Khennak, I., Drias, H., \& Kechid, S. (2016). A New Modeling of Query Expansion Using an Effective BatInspired Optimization Algorithm. IFAC-PapersOnLine, 49(12), 1791-1796. doi:10.1016/j.ifacol.2016.07.842

Kim, S., Fiorini, N., Wilbur, W. J., \& Lu, Z. (2017). Bridging the gap: Incorporating a semantic similarity measure for effectively mapping PubMed queries to documents. Journal of Biomedical Informatics, 75, 122-127. doi:10.1016/j.jbi.2017.09.014 PMID:28986328

Klein, D., \& Manning, C.D. (2007). Accurate unlexicalized parsing. 10.3115/1075096.1075150

Latard, B., Weber, J., Forestier, G., \& Hassenforder, M. (2017). Towards a semantic search engine for scientific articles. Lect. Notes Comput. Sci. (including Subser. Lect. Notes Artif. Intell. Lect. Notes Bioinformatics) 10450 LNCS, 608-611. doi:10.1007/978-3-319-67008-9_54

Lee, C. S., \& Ma, L. (2012). News sharing in social media: The effect of gratifications and prior experience. Computers in Human Behavior, 28(2), 331-339. doi:10.1016/j.chb.2011.10.002 
Levene, M. (2011). Search Engines: Information Retrieval in Practice, The Computer Journal. Addison-Wesley. doi:10.1093/comjnl/bxq039

Mihalcea, R., \& Moldovan, D. (2000). Semantic indexing using WordNet senses. Proceedings of the ACL2000 Workshop on Recent Advances in Natural Language Processing and Information Retrieval: Held in Conjunction with the 38th Annual Meeting of the Association for Computational Linguistics, 11, 35-45. doi:10.3115/1117755.1117760

Miller, G. A. (1995). WordNet: A Lexical Database for English. Communications of the ACM, 38(11), 39-41. doi: $10.1145 / 219717.219748$

Noraset, T., Liang, C., Birnbaum, L., \& Downey, D. (2016). Definition Modeling: Learning to define word embeddings in natural language. Academic Press.

Othman, N., Faiz, R., Smaili, K., Embedding, A.W., Othman, N., Faiz, R., \& Smaili, K. (2017). A Word Embedding based Method for Question Retrieval in Community Question Answering. Academic Press.

Ponte, J. M., \& Croft, W. B. (1998). A language modeling approach to information retrieval. University of Massachusetts at Amherst. doi:10.1145/290941.291008

Porter, M. F. (1980). An algorithm for suffix stripping. Program, 14(3), 130-137. doi:10.1108/eb046814

Porter, M.F. (2001). Snowball: A language for stemming algorithms. Academic Press.

Resnik, P. (1995). Using Information Content to Evaluate Semantic Similarity in a Taxonomy. Academic Press.

Sánchez Fernández, M. (2009). Semantically enhanced Information Retrieval: an ontology-based approach. Academic Press.

Serizawa, M., \& Kobayashi, I. (2013). A study on query expansion based on topic distributions of retrieved documents. International Conference on Intelligent Text Processing and Computational Linguistics, 369-379. doi:10.1007/978-3-642-37256-8_31

Singh, J., \& Sharan, A. (2018). Rank fusion and semantic genetic notion based automatic query expansion model. Swarm and Evolutionary Computation, 38, 295-308. doi:10.1016/j.swevo.2017.09.007

Stein, R. A., Jaques, P. A., \& Valiati, J. F. (2019). An analysis of hierarchical text classification using word embeddings. Inf. Sci. (Ny), 471, 216-232. doi:10.1016/j.ins.2018.09.001

Tablan, V., Bontcheva, K., Roberts, I., \& Cunningham, H. (2015). Mímir: An open-source semantic search framework for interactive information seeking and discovery. Journal of Web Semantics, 30, 52-68. doi:10.1016/j. websem.2014.10.002

Wang, F., Wu, W., Li, Z., \& Zhou, M. (2017). Named entity disambiguation for questions in community question answering. Knowledge-Based Systems, 126, 68-77. doi:10.1016/j.knosys.2017.03.017

Yan, L., Ma, R., Li, D., \& Cheng, J. (2017). RDF approximate queries based on semantic similarity. Computing, 99(5), 481-491. doi:10.1007/s00607-017-0554-9

Zhai, C., Cohen, W. W., \& Lafferty, J. (2015). Beyond independent relevance: methods and evaluation metrics for subtopic retrieval. ACM SIGIR Forum, 2-9. doi:10.1145/2795403.2795405

Zhao, G., Wu, J., Wang, D., \& Li, T. (2016). Entity disambiguation to Wikipedia using collective ranking. Information Processing \& Management, 52(6), 1247-1257. doi:10.1016/j.ipm.2016.06.002

Zhu, X., Huang, J., Zhou, B., Li, A., \& Jia, Y. (2017). Real-time personalized twitter search based on semantic expansion and quality model. Neurocomputing, 254, 13-21. doi:10.1016/j.neucom.2016.10.082

Zingla, M. A., Chiraz, L., \& Slimani, Y. (2016). Short Query Expansion for Microblog Retrieval. Procedia Computer Science, 96, 225-234. doi:10.1016/j.procs.2016.08.135 
Tarek Chenaina is a Software Engineer, Ph.D. in Computer Science, Artificial Intelligence and Form Recognition, University of Paris 6. Associate Professor at Taibah University (2011-2020), Assistant Professor at Tunisian National School of Computer Science (1998-2010). IEEE Computer Society Member. Former Director of the Higher Institute of Informatics and Multimedia of Gabès. President of the Curriculum Committee, the Accreditation Committee, the Training Committee, the e-commerce Committee and the Registration Committee. Administrative coordinator of the Computer Sciences and Information Technology college with the college of Administration Sciences and Planning for Information Systems Programs (King Faisal university \& Taibah University, KSA).

Sameh Neji received her M.Sc. in Software Engineering and Computer Science from Tunisia University, Tunisia in 2013. She was an IT Teacher in United Nations High Commissioner for Refugees (UNHCR) and in "Save The Children" organization, Tunisia. She was a Lecturer in Management Information Systems Department, College of Business Administration, Taibah University, Yanbu, Saudi Arabia during 2014-2018. In Taibah University, She was the Vice Supervisor of the Internship Unit, Vice Supervisor of university counseling unit, Supervisor of educational affairs, Member of the steering committee. Currently, She is a PhD. Candidate in Computer Science, Sfax University. She is interested in Information Retrieval Systems, Semantic Search Engines.

Abdullah M. Shoeb received his Ph.D. in Computer Science from Fayoum University, Egypt in 2009. He is Assistant Professor in Computer Science Department, Faculty of Computers and Information, Fayoum University, Egypt. He had many leadership positions in his college and university like, Manager of Quality Assurance Unit, Manager of "Raising the Efficiency of the Use of Information and Communication Technology in Education" project, Manager of Electronic Courses Production Center (Fayoum Univ.), Member of Faculty of Computers and Information Board, Coordinator of Integrated University Management System Project. He is also a certified trainer of trainers. He was an Assistant Professor in Management Information Systems Department, College of Business Administration, Taibah University, Yanbu, Saudi Arabia during 2014-2018. In Taibah University, He was the Head of Management Information Systems Department, and a supervisor of the Internship Unit and a Supervisor of university counseling unit. 